\title{
Problems of the reference radio source selection
}

\author{
Oleg A. Titov \\ Geoscience Australia, GPO Box 378, Canberra, ACT 2601, Australia \\ email: oleg.titov@ga.gov.au
}

Abstract. We discuss problems of the reference radio source selection.

Keywords. data analysis, quasars: general, reference systems

The International Celestial Reference Frame (ICRF) is realised by high precision coordinates of the extragalactic radio sources observed by VLBI. Only radio sources with stable positions should be used in the ICRF catalogue to maintain long-term stability of the reference system. However, some radio sources previously treated as astrometrically stable, show significant variations in position on different time scales. In this paper the positional instabilities of selected radio sources are discussed. It is shown that apparent proper motions for frequently observed objects can reach 1 mas/yr over several years. Therefore, more careful procedure of the reference radio source selection should be applied.

Several global solutions based on different sets of reference radio sources have also been obtained using OCCAM software by the least squares collocation method. It was shown that the existing lists of reference radio sources produce some controversial results. These solution statistics and resulted astrometric position catalogues were investigated to draw conclusion about more effective scheme for the reference radio source selection. 